\title{
On an Algorithmic Algebra over Simple-Named Complex-Valued Nominative Data
}

\author{
Ievgen Ivanov \\ Taras Shevchenko National University \\ Kyiv, Ukraine
}

\author{
Artur Korniłowicz \\ Institute of Informatics \\ University of Białystok \\ Poland
}

\author{
Mykola Nikitchenkc \\ Taras Shevchenko National University \\ Kyiv, Ukraine
}

Summary. This paper continues formalization in the Mizar system 2, 1] of basic notions of the composition-nominative approach to program semantics 14] which was started in [8, 12, 10].

The composition-nominative approach studies mathematical models of computer programs and data on various levels of abstraction and generality and provides tools for reasoning about their properties. In particular, data in computer systems are modeled as nominative data [15. Besides formalization of semantics of programs, certain elements of the composition-nominative approach were applied to abstract systems in a mathematical systems theory [4, 6, 7, 5, 3].

In the paper we give a formal definition of the notions of a binominative function over given sets of names and values (i.e. a partial function which maps simple-named complex-valued nominative data to such data) and a nominative predicate (a partial predicate on simple-named complex-valued nominative data). The sets of such binominative functions and nominative predicates form the carrier of the generalized Glushkov algorithmic algebra for simple-named complex-valued nominative data [15. This algebra can be used to formalize algorithms which operate on various data structures (such as multidimensional arrays, lists, etc.) and reason about their properties.

In particular, we formalize the operations of this algebra which require a specification of a data domain and which include the existential quantifier, the assignment composition, the composition of superposition into a predicate, the composition of superposition into a binominative function, the name checking 
predicate. The details on formalization of nominative data and the operations of the algorithmic algebra over them are described in [11, 13, 9].

MSC: 68Q60 68T37 03B70 03B35

Keywords: Glushkov algorithmic algebra; nominative data

MML identifier: NOMIN_2, version: $8.1 .08 \quad 5.52 .1328$

\section{Preliminaries}

From now on $a, b, c, v, v_{1}, x, y$ denote objects, $V, A$ denote sets, and $d$ denotes a nominative data with simple names from $V$ and complex values from $A$.

Now we state the proposition:

(1) $\{a, b, c\} \subseteq A$ if and only if $a, b, c \in A$.

Let $a, b, c, d, e, f$ be objects. One can verify that $\{\langle a, b\rangle,\langle c, d\rangle,\langle e, f\rangle\}$ is relation-like.

Let us consider objects $a, b, c, d, e, f$. Now we state the propositions:

(2) $\operatorname{dom}\{\langle a, b\rangle,\langle c, d\rangle,\langle e, f\rangle\}=\{a, c, e\}$.

(3) $\operatorname{rng}\{\langle a, b\rangle,\langle c, d\rangle,\langle e, f\rangle\}=\{b, d, f\}$.

Let us consider $V$. Note that there exists a finite sequence which is one-to-one and $V$-valued.

(4) $\operatorname{dom}\langle a, b, c\rangle=\{1,2,3\}$.

Let us consider $V$ and $A$. Let us note that $\operatorname{ND}_{\mathrm{SS}}(V, A)$ is non empty and has not non empty elements and $\mathrm{ND}_{\mathrm{SC}}(V, A)$ is non empty and has not non empty elements.

Now we state the propositions:

(5) If $v \in V$, then $\{\langle v, d\rangle\}$ is a non-atomic nominative data of $V$ and $A$.

(6) Let us consider a finite function $D$. Suppose $\operatorname{dom} D \subseteq V$ and $\operatorname{rng} D \subseteq$ $\mathrm{ND}_{\mathrm{SC}}(V, A)$. Then $D$ is a non-atomic nominative data of $V$ and $A$.

Proof: Define $\mathcal{P}[$ set $] \equiv \$_{1}$ is a non-atomic nominative data of $V$ and $A$. For every sets $x, B$ such that $x \in D$ and $B \subseteq D$ and $\mathcal{P}[B]$ holds $\mathcal{P}[B \cup\{x\}]$. $\mathcal{P}[D]$.

(7) Let us consider nominative data $d_{1}, d_{2}$ with simple names from $V$ and complex values from $A$. Then $d_{2} \subseteq d_{1} \nabla_{a} d_{2}$.

(8) Every non-atomic nominative data of $V$ and $A$ is a nominative data with simple names from $V$ and complex values from $A$. 
(9) Let us consider non-atomic nominative data $d_{1}, d_{2}$ of $V$ and $A$. Then $d_{1} \nabla_{a} d_{2}$ is a non-atomic nominative data of $V$ and $A$. The theorem is a consequence of (8).

Let us consider $V$ and $A$. Let $d_{1}, d_{2}$ be non-atomic nominative data of $V$ and $A$. Let us observe that $d_{1} \nabla_{a} d_{2}$ is function-like and relation-like.

Let us consider $v$. One can verify that $d_{1} \nabla_{a}^{v} d_{2}$ is function-like and relationlike.

Let $d_{1}$ be a non-atomic nominative data of $V$ and $A$ and $d_{2}$ be a nominative data with simple names from $V$ and complex values from $A$. Let us observe that $d_{1} \nabla_{a}^{v} d_{2}$ is function-like and relation-like.

Now we state the propositions:

(10) Suppose $v \in V$. Let us consider nominative data $d_{1}, d_{2}$ with simple names from $V$ and complex values from $A$, and a function $L$. If $L=d_{1} \nabla_{a}^{v} d_{2}$, then $L(v)=d_{2}$. The theorem is a consequence of (8).

(11) Suppose $v \in V$ and $v \neq v_{1}$. Let us consider a non-atomic nominative data $d_{1}$ of $V$ and $A$, a nominative data $d_{2}$ with simple names from $V$ and complex values from $A$, and a function $L$. Suppose $L=d_{1} \nabla_{a}^{v} d_{2}$ and $v_{1} \in \operatorname{dom} d_{1}$ and $d_{1} \notin A$ and $\Rightarrow v\left(d_{2}\right) \notin A$. Then $L\left(v_{1}\right)=d_{1}\left(v_{1}\right)$. The theorem is a consequence of (8).

Let us consider a non-atomic nominative data $d_{1}$ of $V$ and $A$ and a nominative data $d_{2}$ with simple names from $V$ and complex values from $A$. Now we state the propositions:

(12) Suppose $v \in V$ and $v \notin \operatorname{dom} d_{1}$ and $d_{1} \notin A$ and $\Rightarrow v\left(d_{2}\right) \notin A$. Then $\operatorname{dom}\left(d_{1} \nabla_{a}^{v} d_{2}\right)=\{v\} \cup \operatorname{dom} d_{1}$.

(13) If $v \in V$ and $v \in \operatorname{dom} d_{1}$ and $d_{1} \notin A$ and $\Rightarrow v\left(d_{2}\right) \notin A$, then $\operatorname{dom}\left(d_{1} \nabla_{a}^{v} d_{2}\right)=$ $\operatorname{dom} d_{1}$.

(14) If $v \in V$ and $d_{1} \notin A$ and $\Rightarrow v\left(d_{2}\right) \notin A$, then $\operatorname{dom}\left(d_{1} \nabla_{a}^{v} d_{2}\right)=\{v\} \cup \operatorname{dom} d_{1}$. The theorem is a consequence of (13) and (12).

Let us consider $V$ and $A$.

A partial predicate over simple-named complex-valued nominative data of $V$ and $A$ is a partial predicate of $\operatorname{ND}_{\mathrm{SC}}(V, A)$. In the sequel $p, q, r$ denote partial predicates over simple-named complex-valued nominative date of $V$ and $A$.

Now we state the propositions:

(15) $\operatorname{dom}(p \vee q)=\{d$, where $d$ is a nominative data with simple names from $V$ and complex values from $A: d \in \operatorname{dom} p$ and $p(d)=$ true or $d \in \operatorname{dom} q$ and $q(d)=$ true or $d \in \operatorname{dom} p$ and $p(d)=$ false and $d \in \operatorname{dom} q$ and $q(d)=$ false $\}$.

(16) $\operatorname{dom}(p \wedge q)=\{d$, where $d$ is a nominative data with simple names from $V$ and complex values from $A: d \in \operatorname{dom} p$ and $p(d)=$ false or $d \in$ 
$\operatorname{dom} q$ and $q(d)=$ false or $d \in \operatorname{dom} p$ and $p(d)=$ true and $d \in \operatorname{dom} q$ and $q(d)=$ true $\}$.

(17) $\operatorname{dom}(p \Rightarrow q)=\{d$, where $d$ is a nominative data with simple names from $V$ and complex values from $A: d \in \operatorname{dom} p$ and $p(d)=$ false or $d \in$ $\operatorname{dom} q$ and $q(d)=$ true or $d \in \operatorname{dom} p$ and $p(d)=$ true and $d \in \operatorname{dom} q$ and $q(d)=$ false $\}$.

Let us consider $V, A$, and $v$. The functor $\exists_{v}^{V, A}$ yielding a function from $\operatorname{Pr}\left(\mathrm{ND}_{\mathrm{SC}}(V, A)\right)$ into $\operatorname{Pr}\left(\mathrm{ND}_{\mathrm{SC}}(V, A)\right)$ is defined by

(Def. 1) for every partial predicate over simple-named complex-valued nominative data $p$ of $V$ and $A, \operatorname{dom}(i t(p))=\{d$, where $d$ is a nominative data with simple names from $V$ and complex values from $A$ : there exists a nominative data $d_{1}$ with simple names from $V$ and complex values from $A$ such that $d \nabla_{a}^{v} d_{1} \in \operatorname{dom} p$ and $p\left(d \nabla_{a}^{v} d_{1}\right)=$ true or for every nominative data $d_{1}$ with simple names from $V$ and complex values from $A, d \nabla_{a}^{v} d_{1} \in$ $\operatorname{dom} p$ and $p\left(d \nabla_{a}^{v} d_{1}\right)=$ false $\}$ and for every nominative data $d$ with simple names from $V$ and complex values from $A$, if there exists a nominative data $d_{1}$ with simple names from $V$ and complex values from $A$ such that $d \nabla_{a}^{v} d_{1} \in \operatorname{dom} p$ and $p\left(d \nabla_{a}^{v} d_{1}\right)=$ true, then $i t(p)(d)=$ true and if for every nominative data $d_{1}$ with simple names from $V$ and complex values from $A, d \nabla_{a}^{v} d_{1} \in \operatorname{dom} p$ and $p\left(d \nabla_{a}^{v} d_{1}\right)=$ false, then it $(p)(d)=$ false.

Let us consider $p$. The functor $\exists_{v} p$ yielding a partial predicate over simplenamed complex-valued nominative data of $V$ and $A$ is defined by the term

(Def. 2) $\quad\left(\exists_{v}^{V, A}\right)(p)$.

Now we state the propositions:

(18) Suppose $x \in \operatorname{dom}\left(\exists_{v} p\right)$. Then

(i) there exists a nominative data $d_{1}$ with simple names from $V$ and complex values from $A$ such that $x \nabla_{a}^{v} d_{1} \in \operatorname{dom} p$ and $p\left(x \nabla_{a}^{v} d_{1}\right)=$ true, or

(ii) for every nominative data $d_{1}$ with simple names from $V$ and complex values from $A, x \nabla_{a}^{v} d_{1} \in \operatorname{dom} p$ and $p\left(x \nabla_{a}^{v} d_{1}\right)=$ false.

(19) $\exists_{v} \perp_{\mathrm{PP}}\left(\mathrm{ND}_{\mathrm{SC}}(V, A)\right)=\perp_{\mathrm{PP}}\left(\mathrm{ND}_{\mathrm{SC}}(V, A)\right)$. The theorem is a consequence of (18).

(20) Distributivity LaW:

$\exists_{v}(p \vee q)=\exists_{v} p \vee \exists_{v} q$. 
2. On an Algorithmic algebra over Simple-Named Complex-Valued Nominative Data

From now on $n$ denotes a natural number and $X$ denotes a function.

Let $F$ be a function yielding function and $d$ be an object. We say that $d$ is in doms $F$ if and only if

(Def. 3) for every object $x$ such that $x \in \operatorname{dom} F$ holds $d \in \operatorname{dom}(F(x))$.

Let $g$ be a function yielding function and $X$ be a function. The functor $\operatorname{NDdataSeq}(g, X, d)$ yielding a function is defined by

(Def. 4) $\operatorname{dom} i t=\operatorname{dom} X$ and for every $x$ such that $x \in \operatorname{dom} X$ holds $i t(x)=$ $\langle X(x), g(x)(d)\rangle$.

Let $X$ be a finite function. Let us note that $\operatorname{NDdataSeq}(g, X, d)$ is finite.

Let $X$ be a finite sequence. One can check that $\operatorname{NDdataSeq}(g, X, d)$ is finite sequence-like.

Let $X$ be a function. The functor $\operatorname{NDentry}(g, X, d)$ yielding a set is defined by the term

(Def. 5) $\operatorname{rng} \operatorname{NDdataSeq}(g, X, d)$.

Now we state the propositions:

(21) Let us consider a function $f$, and objects $a, d$. Then NDentry $(\langle f\rangle,\langle a\rangle, d)=$ $\{\langle a, f(d)\rangle\}$.

(22) Let us consider functions $f, g$, and objects $a, b, d$. Then $\operatorname{NDentry}(\langle f$, $g\rangle,\langle a, b\rangle, d)=\{\langle a, f(d)\rangle,\langle b, g(d)\rangle\}$.

(23) Let us consider functions $f, g, h$, and objects $a, b, c, d$. Then NDentry $(\langle f$, $g, h\rangle,\langle a, b, c\rangle, d)=\{\langle a, f(d)\rangle,\langle b, g(d)\rangle,\langle c, h(d)\rangle\}$. The theorem is a consequence of (4).

Let $g$ be a function yielding function, $X$ be a function, and $d$ be an object. Let us note that $\operatorname{NDentry}(g, X, d)$ is relation-like.

Let $X$ be a one-to-one function. One can verify that $\operatorname{NDentry}(g, X, d)$ is function-like.

Let $X$ be a finite function. Observe that $\operatorname{NDentry}(g, X, d)$ is finite.

Now we state the proposition:

(24) Let us consider a function yielding function $g$, a function $X$, and an object $d$. Then $\operatorname{dom}(\operatorname{NDentry}(g, X, d))=\operatorname{rng} X$.

Let us consider $V$ and $A$.

A binominative function over simple-named complex-valued nominative data of $V$ and $A$ is a partial function from $\operatorname{ND}_{\mathrm{SC}}(V, A)$ to $\operatorname{ND}_{\mathrm{SC}}(V, A)$. From now on $f, g, h$ denote binominative functions over simple-named complex-valued nominative date of $V$ and $A$. 
Now we state the propositions:

(25) $\operatorname{rng} \operatorname{NDdataSeq}(\langle f\rangle,\langle v\rangle, d)=v \longmapsto f(d)$.

(26) If $a \in V$ and $d \in \operatorname{dom} f$, then $\operatorname{NDentry}(\langle f\rangle,\langle a\rangle, d)=\Rightarrow a(f(d))$. The theorem is a consequence of (25).

(27) If $a \in V$ and $d \in \operatorname{dom} f$, then $\operatorname{NDentry}(\langle f\rangle,\langle a\rangle, d)$ is a non-atomic nominative data of $V$ and $A$. The theorem is a consequence of (26).

(28) Suppose $\{a, b\} \subseteq V$ and $a \neq b$ and $d \in \operatorname{dom} f$ and $d \in \operatorname{dom} g$. Then NDentry $(\langle f, g\rangle,\langle a, b\rangle, d)$ is a non-atomic nominative data of $V$ and $A$. The theorem is a consequence of (22) and (6).

(29) Suppose $\{a, b, c\} \subseteq V$ and $a, b, c$ are mutually different and $d \in \operatorname{dom} f$ and $d \in \operatorname{dom} g$ and $d \in \operatorname{dom} h$. Then NDentry $(\langle f, g, h\rangle,\langle a, b, c\rangle, d)$ is a nonatomic nominative data of $V$ and $A$. The theorem is a consequence of (23), (2), (3), (1), and (6).

Let us consider $V$ and $A$. Let $f$ be a finite sequence. We say that $f$ is $(V, A)$-FPrg-yielding if and only if

(Def. 6) for every $n$ such that $1 \leqslant n \leqslant \operatorname{len} f$ holds $f(n)$ is a binominative function over simple-named complex-valued nominative data of $V$ and $A$.

Let us consider $f$. Let us note that $\langle f\rangle$ is $(V, A)$-FPrg-yielding.

Let us consider $g$. Note that $\langle f, g\rangle$ is $(V, A)$-FPrg-yielding.

Let us consider $h$. Let us observe that $\langle f, g, h\rangle$ is $(V, A)$-FPrg-yielding.

Let us consider $n$. One can verify that there exists a finite sequence which is $(V, A)$-FPrg-yielding and $n$-element.

Let us consider $x$. Let $g$ be a $(V, A)$-FPrg-yielding finite sequence. One can verify that $g(x)$ is function-like and relation-like and every finite sequence which is $(V, A)$-FPrg-yielding is also function yielding.

Now we state the propositions:

(30) Let us consider a $(V, A)$-FPrg-yielding finite sequence $g$, and a one-toone finite sequence $X$. Suppose $\operatorname{dom} g=\operatorname{dom} X$ and $d$ is in doms $g$. Then $\operatorname{rng} \operatorname{NDentry}(g, X, d) \subseteq \operatorname{ND}_{\mathrm{SC}}(V, A)$.

(31) Let us consider a $(V, A)$-FPrg-yielding finite sequence $g$, and a one-toone, $V$-valued finite sequence $X$. Suppose $\operatorname{dom} g=\operatorname{dom} X$ and $d$ is in doms $g$. Then $\operatorname{NDentry}(g, X, d)$ is a non-atomic nominative data of $V$ and $A$. The theorem is a consequence of (24), (30), and (6).

Let us consider $V, A$, and $v$. The functor $\mathrm{Asg}^{V, A, v}$ yielding a function from $\operatorname{FPrg}\left(\mathrm{ND}_{\mathrm{SC}}(V, A)\right)$ into $\operatorname{FPrg}\left(\mathrm{ND}_{\mathrm{SC}}(V, A)\right)$ is defined by

(Def. 7) for every binominative function over simple-named complex-valued nominative data $f$ of $V$ and $A, \operatorname{dom}(i t(f))=\operatorname{dom} f$ and for every nominative 
data $d$ with simple names from $V$ and complex values from $A$ such that $d \in \operatorname{dom}(i t(f))$ holds $i t(f)(d)=d \nabla_{a}^{v} f(d)$.

Let us consider $V, A, v$, and $f$. The functor $\operatorname{Asg}^{v}(f)$ yielding a binominative function over simple-named complex-valued nominative data of $V$ and $A$ is defined by the term

(Def. 8) $\operatorname{Asg}^{V, A, v}(f)$.

Let $d$ be a non-atomic nominative data of $V$ and $A$. One can check that $\operatorname{Asg}^{v}(f)(d)$ is function-like and relation-like.

Now we state the proposition:

(32) Let us consider a non-atomic nominative data $d$ of $V$ and $A$. Suppose $v \in V$ and $d \notin A$ and $\Rightarrow v(f(d)) \notin A$ and $d \in \operatorname{dom} f$. Then $\operatorname{dom}\left(\left(\operatorname{Asg}^{v}(f)\right)(d)\right)=\operatorname{dom} d \cup\{v\}$. The theorem is a consequence of (14).

Let us consider $V$ and $A$. Let $g$ be a $(V, A)$-FPrg-yielding finite sequence. Assume $\prod g \neq \emptyset$. Let $X$ be a function. The functor $\operatorname{SP}_{\mathrm{P}}(g, X)$ yielding a function from $\operatorname{Pr}\left(\mathrm{ND}_{\mathrm{SC}}(V, A)\right) \times \prod g$ into $\operatorname{Pr}\left(\mathrm{ND}_{\mathrm{SC}}(V, A)\right)$ is defined by

(Def. 9) for every partial predicate over simple-named complex-valued nominative data $p$ of $V$ and $A$ and for every element $x$ of $\prod g, \operatorname{dom} i t(p, x)=$ $\{d$, where $d$ is a nominative data with simple names from $V$ and complex values from $A: d \nabla_{a}(\operatorname{NDentry}(g, X, d)) \in \operatorname{dom} p$ and $d$ is in doms $\left.g\right\}$ and for every nominative data $d$ with simple names from $V$ and complex values from $A$ such that $d$ is in doms $g$ holds $i t(p, x)(d) \cong p\left(d \nabla_{a}(\operatorname{NDentry}(g, X, d))\right)$.

Let us consider $V, A$, and $p$. Let $g$ be a $(V, A)$-FPrg-yielding finite sequence. Assume $\prod g \neq \emptyset$. Let $X$ be a function and $x$ be an element of $\prod g$. The functor $\mathrm{S}_{\mathrm{P}}(p, x, X)$ yielding a partial predicate over simple-named complex-valued nominative data of $V$ and $A$ is defined by the term

(Def. 10) $\mathrm{S}_{\mathrm{P}}(g, X)(p, x)$.

Now we state the proposition:

(33) Let us consider a $(V, A)$-FPrg-yielding finite sequence $g$. Suppose $\prod g \neq$ $\emptyset$. Let us consider an element $x$ of $\prod g$. Suppose $d \in \operatorname{dom}\left(\mathrm{S}_{\mathrm{P}}(p, x, X)\right)$. Then

(i) $d$ is in doms $g$, and

(ii) $\mathrm{S}_{\mathrm{P}}(p, x, X)(d)=p\left(d \nabla_{a}(\operatorname{NDentry}(g, X, d))\right)$.

Let us consider $V, A$, and $v$. The functor $\mathrm{S}_{\mathrm{P}}^{V, A, v}$ yielding a function from $\operatorname{Pr}\left(\mathrm{ND}_{\mathrm{SC}}(V, A)\right) \times \operatorname{FPrg}\left(\mathrm{ND}_{\mathrm{SC}}(V, A)\right)$ into $\operatorname{Pr}\left(\mathrm{ND}_{\mathrm{SC}}(V, A)\right)$ is defined by

(Def. 11) for every partial predicate over simple-named complex-valued nominative data $p$ of $V$ and $A$ and for every binominative function over simplenamed complex-valued nominative data $f$ of $V$ and $A, \operatorname{dom} i t(p, f)=$ $\{d$, where $d$ is a nominative data with simple names from $V$ and complex 
values from $A: d \nabla_{a}^{v} f(d) \in \operatorname{dom} p$ and $\left.d \in \operatorname{dom} f\right\}$ and for every nominative data $d$ with simple names from $V$ and complex values from $A$ such that $d \in \operatorname{dom} f$ holds $i t(p, f)(d) \cong p\left(d \nabla_{a}^{v} f(d)\right)$.

Let us consider $V, A, v, p$, and $f$. The functor $\mathrm{S}_{\mathrm{P}}(p, f, v)$ yielding a partial predicate over simple-named complex-valued nominative data of $V$ and $A$ is defined by the term

(Def. 12) $\quad\left(\mathrm{S}_{\mathrm{P}}^{V, A, v}\right)(p, f)$.

Now we state the propositions:

(34) If $d \in \operatorname{dom}\left(\mathrm{S}_{\mathrm{P}}(p, f, v)\right)$, then $\mathrm{S}_{\mathrm{P}}(p, f, v)(d)=p\left(d \nabla_{a}^{v} f(d)\right)$ and $d \in \operatorname{dom} f$.

(35) Let us consider an element $x$ of $\prod\langle f\rangle$. Suppose $v \in V$ and $\prod\langle f\rangle \neq \emptyset$. Then $\mathrm{S}_{\mathrm{P}}(p, f, v)=\mathrm{S}_{\mathrm{P}}(p, x,\langle v\rangle)$. The theorem is a consequence of (26), (33), and (34).

Let us consider $V$ and $A$. Let $g$ be a $(V, A)$-FPrg-yielding finite sequence. Assume $\prod g \neq \emptyset$. Let $X$ be a function. The functor $\mathrm{S}_{\mathrm{F}}(g, X)$ yielding a function from $\operatorname{FPrg}\left(\mathrm{ND}_{\mathrm{SC}}(V, A)\right) \times \prod g$ into $\operatorname{FPrg}\left(\mathrm{ND}_{\mathrm{SC}}(V, A)\right)$ is defined by

(Def. 13) for every binominative function over simple-named complex-valued nominative data $f$ of $V$ and $A$ and for every element $x$ of $\prod g$, dom it $(f, x)=$ $\{d$, where $d$ is a nominative data with simple names from $V$ and complex values from $A: d \nabla_{a}(\operatorname{NDentry}(g, X, d)) \in \operatorname{dom} f$ and $d$ is in doms $\left.g\right\}$ and for every nominative data $d$ with simple names from $V$ and complex values from $A$ such that $d$ is in doms $g$ holds $i t(f, x)(d) \cong f\left(d \nabla_{a}(\operatorname{NDentry}(g, X, d))\right)$.

Let us consider $V, A$, and $f$. Let $g$ be a $(V, A)$-FPrg-yielding finite sequence. Assume $\prod g \neq \emptyset$. Let $X$ be a function and $x$ be an element of $\prod g$. The functor $\mathrm{S}_{\mathrm{F}}(f, x, X)$ yielding a binominative function over simple-named complex-valued nominative data of $V$ and $A$ is defined by the term

(Def. 14) $\mathrm{S}_{\mathrm{F}}(g, X)(f, x)$.

Now we state the proposition:

(36) Let us consider a $(V, A)$-FPrg-yielding finite sequence $g$. Suppose $\prod g \neq$ $\emptyset$. Let us consider an element $x$ of $\prod g$. Suppose $d \in \operatorname{dom}\left(\mathrm{S}_{\mathrm{F}}(f, x, X)\right)$. Then

(i) $d$ is in doms $g$, and

(ii) $\mathrm{S}_{\mathrm{F}}(f, x, X)(d)=f\left(d \nabla_{a}(\operatorname{NDentry}(g, X, d))\right)$.

Let us consider $V, A$, and $v$. The functor $\mathrm{S}_{\mathrm{F}}^{V, A, v}$ yielding a function from $\operatorname{FPrg}\left(\mathrm{ND}_{\mathrm{SC}}(V, A)\right) \times \operatorname{FPrg}\left(\mathrm{ND}_{\mathrm{SC}}(V, A)\right)$ into $\mathrm{FPrg}\left(\mathrm{ND}_{\mathrm{SC}}(V, A)\right)$ is defined by

(Def. 15) for every binominative functions over simple-named complex-valued nominative date $f, g$ of $V$ and $A$, dom it $(f, g)=\{d$, where $d$ is a nominative data with simple names from $V$ and complex values from $A: d \nabla_{a}^{v} g(d) \in$ 
$\operatorname{dom} f$ and $d \in \operatorname{dom} g\}$ and for every nominative data $d$ with simple names from $V$ and complex values from $A$ such that $d \in \operatorname{dom} g$ holds $i t(f, g)(d) \cong f\left(d \nabla_{a}^{v} g(d)\right)$.

Let us consider $V, A, v, f$, and $g$. The functor $\mathrm{S}_{\mathrm{F}}(f, g, v)$ yielding a binominative function over simple-named complex-valued nominative data of $V$ and $A$ is defined by the term

(Def. 16) $\quad\left(\mathrm{S}_{\mathrm{F}}^{V, A, v}\right)(f, g)$.

Now we state the propositions:

(37) If $d \in \operatorname{dom}\left(\mathrm{S}_{\mathrm{F}}(f, g, v)\right)$, then $\mathrm{S}_{\mathrm{F}}(f, g, v)(d)=f\left(d \nabla_{a}^{v} g(d)\right)$ and $d \in \operatorname{dom} g$.

(38) Let us consider an element $x$ of $\prod\langle g\rangle$. Suppose $v \in V$ and $\prod\langle g\rangle \neq \emptyset$. Then $\mathrm{S}_{\mathrm{F}}(f, g, v)=\mathrm{S}_{\mathrm{F}}(f, x,\langle v\rangle)$. The theorem is a consequence of (26), (36), and (37).

Let us consider $V, A$, and $v$. The functor $v !^{V, A}$ yielding a partial predicate over simple-named complex-valued nominative data of $V$ and $A$ is defined by

(Def. 17) $\operatorname{dom} i t=\mathrm{ND}_{\mathrm{SC}}(V, A) \backslash A$ and for every non-atomic nominative data $d$ of $V$ and $A$ such that $d \in \operatorname{dom}$ it holds if $v \Rightarrow_{a} d \in \operatorname{dom} i t$, then $i t(d)=$ true and if $v \Rightarrow_{a} d \notin \operatorname{dom} i t$, then $i t(d)=$ false.

\section{REFERENCES}

[1] Grzegorz Bancerek, Czesław Byliński, Adam Grabowski, Artur Korniłowicz, Roman Matuszewski, Adam Naumowicz, and Karol Pąk. The role of the Mizar Mathematical Library for interactive proof development in Mizar Journal of Automated Reasoning, 61(1):9-32, 2018. do1:10.1007/s10817-017-9440-6

[2] Adam Grabowski, Artur Korniłowicz, and Adam Naumowicz. Four decades of Mizar. Journal of Automated Reasoning, 55(3):191-198, 2015. doi 10.1007/s10817-015-9345-1

[3] Ievgen Ivanov. On the underapproximation of reach sets of abstract continuous-time systems In Erika Ábrahám and Sergiy Bogomolov, editors, Proceedings 3rd International Workshop on Symbolic and Numerical Methods for Reachability Analysis, SNR@ETAPS 2017. Uppsala, Sweden, 22nd April 2017, volume 247 of EPTCS, pages 46-51, 2017. doi:10.4204/EPTCS.247.4.

[4] Ievgen Ivanov. On representations of abstract systems with partial inputs and outputs. In T. V. Gopal, Manindra Agrawal, Angsheng Ll, and S. Barry Cooper, editors, Theory and Applications of Models of Computation - 11th Annual Conference, TAMC 2014, Chennai, India, April 11-13, 2014. Proceedings, volume 8402 of Lecture Notes in Computer Science, pages 104-123. Springer, 2014. ISBN 978-3-319-06088-0. doi 10.1007/978-3-319-06089-7_8.

[5] Ievgen Ivanov. On local characterization of global timed bisimulation for abstract continuous-time systems. In Ichiro Hasuo, editor, Coalgebraıc Methods in Computer Scıence - 13th IFIP WG 1.3 International Workshop, CMCS 2016, Colocated with ETAPS 2016, Eindhoven, The Netherlands, April 2-3, 2016, Revised Selected Papers, volume 9608 of Lecture Notes in Computer Science, pages 216-234. Springer, 2016. ISBN 978-3319-40369-4. doi 10.1007/978-3-319-40370-0_13.

[6] Ievgen Ivanov, Mykola Nikitchenko, and Uri Abraham. On a decidable formal theory for abstract continuous-time dynamical systems. In Vadim Ermolayev, Heinrich C. Mayr, Mykola Nikitchenko, Aleksander Spivakovsky, and Grygoriy Zholtkevych, editors, Information and Communication Technologies in Education, Research, and Industrial Applications: 10th International Conference, ICTERI 2014, Kherson, Ukraine, June 9-12, 2014, 
Revised Selected Papers, pages 78-99. Springer International Publishing, 2014. ISBN 978-3-319-13206-8. doi 10.1007/978-3-319-13206-8_4.

[7] Ievgen Ivanov, Mykola Nikitchenko, and Uri Abraham. Event-based proof of the mutual exclusion property of Peterson's algorithm. Formalized Mathematics, 23(4):325-331, 2015. doi: $10.1515 /$ forma-2015-0026.

[8] Ievgen Ivanov, Mykola Nikitchenko, Andrii Kryvolap, and Artur Korniłowicz. Simplenamed complex-valued nominative data - definition and basic operations. Formalized Mathematics, 25(3):205-216, 2017. doi 10.1515/forma-2017-0020

[9] Ievgen Ivanov, Artur Korniłowicz, and Mykola Nikitchenko. Implementation of the composition-nominative approach to program formalization in Mizar. The Computer Science Journal of Moldova, 26(1):59-76, 2018.

[10] Ievgen Ivanov, Artur Korniłowicz, and Mykola Nikitchenko. On algebras of algorithms and specifications over uninterpreted data. Formalized Mathematics, 26(2):141-147, 2018. doi:10.2478/forma-2018-0011

[11] Artur Kornilowicz, Andrii Kryvolap, Mykola Nikitchenko, and Ievgen Ivanov. Formalization of the algebra of nominative data in Mizar In Maria Ganzha, Leszek A. Maciaszek, and Marcin Paprzycki, editors, Proceedings of the 2017 Federated Conference on Computer Science and Information Systems, FedCSIS 2017, Prague, Czech Republic, September 3-6, 2017., pages 237-244, 2017. ISBN 978-83-946253-7-5. doi:10.15439/2017F301

[12] Artur Korniłowicz, Ievgen Ivanov, and Mykola Nikitchenko. Kleene algebra of partial predicates. Formalized Mathematics, 26(1):11-20, 2018. doi:10.2478/forma-2018-0002

[13] Artur Korniłowicz, Andrii Kryvolap, Mykola Nikitchenko, and Ievgen Ivanov. Formalization of the nominative algorithmic algebra in Mizar. In Jerzy Świątek, Leszek Borzemski, and Zotia Wilimowska, editors, Information Systems Architecture and Technology: Proceedings of 38th International Conference on Information Systems Architecture and Technology - ISAT 2017: Part II, pages 176-186. Springer International Publishing, 2018. ISBN 978-3-319-67229-8. doi 10.1007/978-3-319-67229-8_16.

[14] Nikolaj S. Nikitchenko. A composition nominative approach to program semantics. Technical Report IT-TR 1998-020, Department of Information Technology, Technical University of Denmark, 1998.

[15] Volodymyr G. Skobelev, Mykola Nikitchenko, and Ievgen Ivanov. On algebraic properties of nominative data and functions. In Vadim Ermolayev, Heinrich C. Mayr, Mykola N1kitchenko, Aleksander Spivakovsky, and Grygoriy Zholtkevych, editors, Information and Communication Technologies in Education, Research, and Industrial Applications - 10th International Conference, ICTERI 2014, Kherson, Ukraine, June 9-12, 2014, Revised Selected Papers, volume 469 of Communications in Computer and Information Science, pages 117-138. Springer, 2014. ISBN 978-3-319-13205-1. doi $10.1007 / 978-3-319-13206-8 \_6$. 